TITLE:

\title{
Diffusion of interface and heat conduction in the three- dimensional Ising model
}

AUTHOR(S):

Masumoto, Yusuke; Takesue, Shinji

\section{CITATION:}

Masumoto, Yusuke ...[et al]. Diffusion of interface and heat conduction in the three-dimensional Ising model. Physical Review E 2019, 99(5): 052128.

ISSUE DATE:

2019-5-20

URL:

http://hdl.handle.net/2433/241561

RIGHT:

(C)2019 American Physical Society 


\title{
Diffusion of interface and heat conduction in the three-dimensional Ising model
}

\author{
Yusuke Masumoto* and Shinji Takesue \\ Department of Physics, Kyoto University, Kyoto 6068502, Japan
}

(Received 28 December 2018; published 20 May 2019)

\begin{abstract}
We investigate the relationship between a diffusive motion of an interface, heat conduction, and the roughening transition in the three-dimensional Ising model. We numerically compute the thermal conductivity and the diffusion constant and find that the diffusion constant shows a crossover in its temperature dependence. The crossover temperature is equal to the roughening transition temperature in equilibrium and deviates from it when heat flows in the system. From these results, we discuss the possibility that heat conduction causes a shift of the roughening transition temperature.
\end{abstract}

DOI: 10.1103/PhysRevE.99.052128

\section{INTRODUCTION}

Dynamical properties of an interface are being studied in relation to various physical phenomena such as crystal growth [1], a grain boundary [2], and a magnetic domain wall. Recently, inspired by the discovery of the spin Seebeck effect, the interface motion induced by a temperature gradient was examined theoretically [3] and experimentally [4]. In both cases, an interface moves to the hotter part of the system. According to the theoretical studies using the stochastic LandauLifshitz-Gilbert and Landau-Lifshitz-Bloch equations [3], this is due to a magnonic spin current and the conservation of angular momentum. Assuming local equilibrium, however, we can explain the result using a thermodynamic argument $[3,5]$. Let us define the free energy of an interface as the difference between the free energy of a system with an interface and that of the same system without an interface. It becomes a monotonically decreasing function of temperature and vanishes at $T_{c}$. Then the interface moves towards the hotter region to minimize the free energy. Since this explanation is quite general, it should be applicable to a wide range of systems with an interface.

In a previous paper [6], we also found that the interface motion in the two-dimensional Ising model is a diffusion process with a drift force towards the high-temperature side, when no magnetic field is applied to the bulk and heat flows in the system. The strength of the drift force is proportional to the difference of temperature values at the two ends. Under an appropriate boundary condition, we prepared the system with an interface and calculated the power spectrum of the temporal sequence of column-averaged magnetizations. It is known that when the step of the step function executes a random walk, the power spectrum of function values at a fixed position shows characteristic power-law behavior with exponent $-3 / 2$ [7]. In equilibrium states of the Ising model with an interface, the column-averaged magnetization shows such a power spectrum with some modification due to the finite

\footnotetext{
*masumoto@scphys.kyoto-u.ac.jp

†takesue@scphys.kyoto-u.ac.jp
}

width of the interface. To simulate heat conduction in the Ising model, we equipped the model with a cellular-automaton type energy-conserving dynamics. We analytically calculated the power spectra in the case where an interface of a width carries out diffusion with a drift. The obtained spectrum showed an excellent agreement with numerical results for the heat conduction states. The thermodynamic explanation can be applied to our case, though not stated in the paper [6]. We calculate the interface energy $\Delta E$ as the difference between the system energy under the antiparallel boundary condition minus that under the parallel one. Then the drag force estimated from the interface free energy $\Delta F(\beta)=\beta^{-1} \int_{0}^{\beta} \Delta E d \beta$ agrees with the numerical results.

To extend our research to three dimensions, we must consider possible influences from the roughening transition. The roughening transition is a phenomenon that a smooth surface turns into a rough one above a certain temperature called the roughening transition temperature. In the Ising model on the simple cubic lattice with isotropic couplings, the roughening transition temperature is $T_{\mathrm{R}}^{\mathrm{eq}}=0.542 T_{c}$ [8], where $T_{c}$ is the critical temperature. At a temperature higher than $T_{\mathrm{R}}^{\mathrm{eq}}$, the interface width is proportional to $\ln L$, where $L$ denotes the system size [8]. It diverges in the thermodynamic limit. In contrast, the interface width is constant for $L \gg 1$ at a temperature lower than $T_{\mathrm{R}}^{\mathrm{eq}}$. The roughness of an interface can affect its motion. Some experiments show that the speed of crystal growth remarkably decreases below the roughening transition temperature $[9,10]$. Thus, it is probable that the diffusion of an interface in the three-dimensional Ising system also shows some changes at the temperature.

In this paper, we focus on how the thermal conductivity and the diffusion coefficient vary near $T_{\mathrm{R}}^{\mathrm{eq}}$ and how heat conduction affects their behavior. In equilibrium, the diffusion constant shows different temperature dependence above and below $T_{\mathrm{R}}^{\mathrm{eq}}$. It decreases more rapidly below $T_{\mathrm{R}}^{\mathrm{eq}}$. For thermal conductivity, the results depend on the time evolution rules for simulations. Thus, we employ two kinds of dynamics and compare those results. Moreover, we examine two kinds of arrangements of an interface. One is an interface perpendicular to heat flow, and the other is an interface parallel to heat flow. 
Most interesting in our results is that when heat flows in the system, the crossover temperature at which the diffusion constant changes temperature dependence deviates from $T_{\mathrm{R}}^{\mathrm{eq}}$. It may indicate that the roughening transition temperature shifts in nonequilibrium situations.

The paper is organized as follows. In Sec. II we define the model and dynamics employed for simulations. In Sec. III we show simulation results on thermal conductivity. In Sec. IV simulation results for diffusion coefficients are exhibited. Section $\mathrm{V}$ is devoted to summary and discussion.

\section{SETUP OF THE SYSTEM}

In the literature, various kinds of spin dynamics have been proposed for the simulation of the Ising model. The most famous one is Glauber dynamics [11], where spins are stochastically updated according to some temperature-dependent transition rates. It is useful for investigating equilibrium properties of the Ising model because the detailed balance condition for the transition rates ensures that the system reaches an equilibrium state at the given temperature. However, it is not appropriate for the simulation of heat conduction, where local temperature values should be determined as a result of heat conduction.

Creutz [12] invented an alternative dynamics that conserves the following Hamiltonian:

$$
H=-\sum_{\langle i, j\rangle} \sigma_{i} \sigma_{j}+\sum_{i} 4 \tilde{\sigma}_{i},
$$

where $\sigma_{i} \in\{-1,+1\}$ denotes the Ising spin on site $i$ and $\tilde{\sigma}_{i} \in$ $\{0,1,2,3\}$ is an auxiliary variable called "momentum." The first term means the usual ferromagnetic interaction, and the second term is a kind of "kinetic energy." In each step, spin $\sigma_{i}$ is flipped if and only if the change in the interaction energy can be compensated by corresponding change of momentum variable $\tilde{\sigma}_{i}$. The condition is written as

$$
0 \leqslant \tilde{\sigma}_{i}-\frac{1}{2} \sigma_{i}\left(\sum_{n n} \sigma_{n n}\right) \leqslant 3,
$$

where $n n$ denotes the nearest-neighbor sites of $i$. If the above inequality is satisfied, spin $\sigma_{i}$ is flipped to $-\sigma_{i}$. Because each momentum obeys the canonical distribution independently of each other, local temperature values can be measured from the distributions or expectation values of momentums. Creutz dynamics was successfully used in the study of heat conduction in the Ising model [13].

A simplified variant of Creutz dynamics is Q2R [14], where the "kinetic energy" term is absent and spins are flipped only if the sum of the nearest-neighbor spins is zero.

In Ref. [6] we have found that Creutz dynamics has a serious problem at low temperature. The interface motion becomes extremely slow and sometimes freezes. Moreover, if the system is attached to a heat reservoir, it does not relax to the uniform equilibrium state at the reservoir temperature within simulation time. Such problems arise from the following reasons. Because most spins are in the same direction below $T_{c}$, a spin flip brings a large increase in the interaction energy. Although a large momentum is necessary to compensate it, it is rare at a low temperature. Thus, the dynamics becomes slow. By the same reason, the thermal conductivity shows a sudden drop around $T_{c}$ [13].

The same problem is noticed in the Q2R, and a solution to the problem was brought by Casartelli et al. [15]. They combined a new dynamics called Kadanoff-Swift dynamics with the Q2R and called the resultant the KQ dynamics. In the $\mathrm{KS}$ dynamics, a pair of next-nearest-neighbor spins exchanges the values if energy is unchanged by the exchange. It should be noted that the Hamiltonian does not have next-nearestneighbor coupling terms. Such spins are only dynamically coupled. By employing the modified dynamics, relaxation to equilibrium was realized in simulation time.

Because we need to measure local temperature values in heat conduction, we modified Creutz dynamics in the similar manner by adding KS dynamics and called the new dynamics Kadanoff-Swift-Creutz (KSC) dynamics in the study of the two-dimensional Ising model [6]. The KSC dynamics also realizes relaxation to equilibrium, and the interface motion does not freeze in the two-dimensional systems.

Microcanonical (MC) dynamics is another spin dynamics that can be used at a low temperature [16]. In the MC dynamics, the "kinetic energy" is defined not on each site but at each bond. At each step, an update of randomly chosen two nearestneighbor spins is considered. We choose a candidate of new configurations for the two spins and calculate the interaction energy variation. If it is compensated by the change of bond momentum, we accept the move. It was originally introduced to simulate a disordered system because the dynamics do not assume a regular lattice structure. It also shows the advantage of high mobility of energy even at a low temperature. In the numerical study in this paper, we employ the KSC dynamics and the MC dynamics and compare the results from the two dynamics.

To simulate heat conduction, the boundary spins in contact with heat reservoirs are evolved by Glauber dynamics. The temperature of the left heat reservoir is denoted by $T_{1}$ and that of the right heat reservoir is by $T_{2}\left(\leqslant T_{1}<T_{c}\right)$. We also use average temperature $T=\left(T_{1}+T_{2}\right) / 2$ and the temperature difference $\Delta T=T_{1}-T_{2}$. Note that we employ an energy unit where the Boltzmann constant is unity and the critical temperature of the three-dimensional Ising model is $1 / T_{c}=$ $0.221654626(5)$ [17]. We can simulate heat conduction using deterministic energy-conserving dynamics such as the KSC dynamics or the MC dynamics for bulk spins. Moreover, if the values of the leftmost ( $x$ direction) and the rightmost spins are fixed to +1 and -1 , respectively, an interface perpendicular to the heat flux is generated between domains with opposite magnetizations. If the values of the top ( $z$ direction) and bottom spins are fixed to +1 and -1 , respectively, an interface parallel to the heat flux is formed. In this paper, we investigate both cases.

\section{THERMAL CONDUCTIVITY}

In this section we present simulation results for the thermal conductivity, which is estimated as

$$
\kappa(T)=J \frac{L_{x}}{\Delta T},
$$




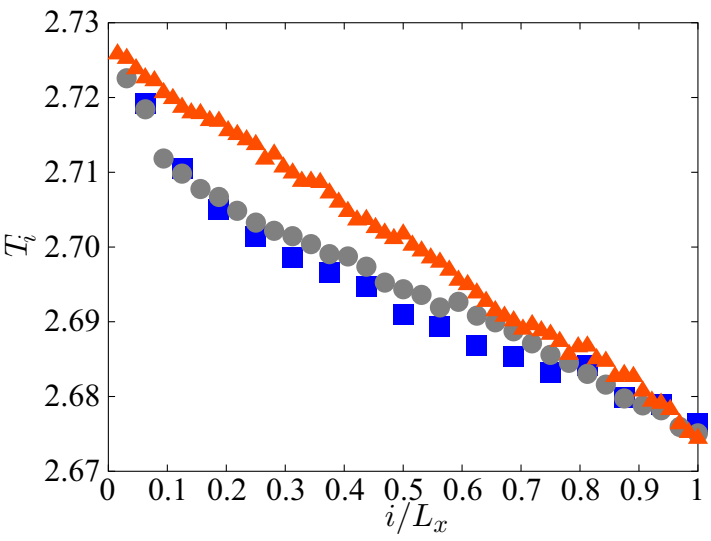

FIG. 1. Temperature profiles for the system with an interface where the KSC dynamics is employed. The system size is $L_{x} \times$ $16 \times 16, L_{x}=16,32,64$, and the reservoir temperatures are $T_{1}=$ 2.725, $T_{2}=2.675$. Blue squares, gray circles, and orange triangles indicate $L_{x}=16,32$, and 64 , respectively.

where $L_{x}$ is the system size in the $x$ direction, $J$ heat flux, and $\Delta T=0.05$. We checked that the result does not seriously change for other choices of $\Delta T$.

First, we deal with the case where the system has an interface perpendicular to the heat flux.

When we employ the KSC dynamics, a finite-size effect is observed in the temperature profile. As seen in Fig. 1, if $L_{x}$ is small, the temperature slope is not uniform, and it is larger in higher temperature region. If $L_{x}$ is greater than or equal to 64 , the finite-size effect vanishes and the uniform temperature gradient is formed. In the following, large enough $L_{x}$ is used not to have the bothering finite-size effect.

In Fig. 2 we compare the thermal conductivity in the system with and without an interface. The thermal conductivity is larger in the system without an interface than in that with an interface as is the case in the two-dimensional system [6]. In $T>T_{\mathrm{R}}^{\mathrm{eq}}$, the thermal conductivity varies like $\kappa(T) \sim$

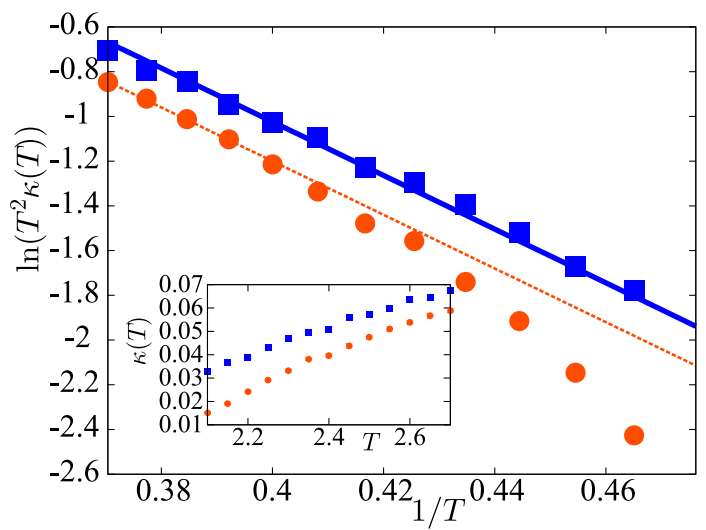

FIG. 2. For a system of size $64 \times 16 \times 16$ developed by the KSC dynamics, $\ln \left[T^{2} \kappa(T)\right]$ is shown as a function of $1 / T$. Inset: plots of $\kappa(T)$ versus $T$. Orange circles and blue squares indicate the system with and without an interface, respectively. Orange dotted line and blue line are guides for the eye to show that $\kappa(T) \sim$ $1 / T^{2} \exp (-12 / T)$ at the high-temperature region.

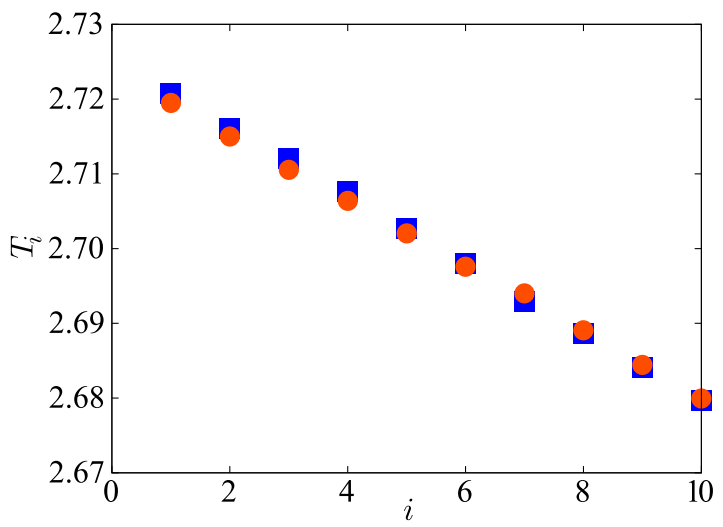

FIG. 3. Temperature profiles generated by MC dynamics. The system size is $10 \times 20 \times 20$, and the reservoir temperatures are $T_{1}=2.725$ and $T_{2}=2.675$. Orange circles and blue squares indicate the system with and without an interface, respectively.

$\frac{1}{T^{2}} \exp (-12 / T)$ in both cases. This temperature dependence is derived from the mean-field-type analysis described in Ref. [13]. In the presence of an interface, the thermal conductivity deviates from the line below $T_{\mathrm{R}}^{\mathrm{eq}}$, where the variation is more rapid than $\exp (-12 / T) / T^{2}$. Such a change in the temperature dependence of $\kappa(T)$ is not observed in the twodimensional systems [6]. Thus, we consider the change in temperature dependence of $\kappa(T)$ near $T_{\mathrm{R}}^{\text {eq }}$ is an effect from the roughening transition. Consider a pair of next-nearestneighbor spins that are located on the opposite sides of a flat interface. To exchange their signs, a large amount of energy is necessary. Thus, the exchange of such spins is virtually inhibited in the KS dynamics. Hence, energy transport through a smooth surface is very difficult at $T<T_{\mathrm{R}}^{\mathrm{eq}}$. This is the reason why the thermal conductivity rapidly decreases below $T_{\mathrm{R}}^{\mathrm{eq}}$.

In contrast to the KSC dynamics, the MC dynamics does not suffer from the finite-size effect seen in the KSC dynamics as seen in Fig. 3. The uniform temperature gradient is realized even in relatively small systems.

Figure 4 show the numerical results of the thermal conductivity in the MC dynamics. Contrary to the KSC dynamics, the thermal conductivity is a little bit smaller in the system without an interface than in that with an interface. Moreover, the thermal conductivity varies like $\kappa(T) \sim \frac{1}{T^{2}} \exp (-12 / T)$ in the whole temperature region. In the MC dynamics, a spin can change its sign only with a variation of a single bond energy. Thus, we consider that the flatness of the interface does not affect the thermal conductivity.

Now we consider the thermal conductivity when the system has an interface parallel to the heat flux using the KSC and $\mathrm{MC}$ dynamics. In this case, there is no noticeable finite-size effects in both the dynamics. Moreover, as seen in Figs. 5 and 6 the mean-field-type temperature dependence can be applied to both dynamics, because energy can transport in the region without an interface.

\section{DIFFUSION CONSTANT}

For the interface perpendicular to heat flux, we observe diffusive motion with a drift to the high-temperature side similar to the two-dimensional case. We find that behavior 


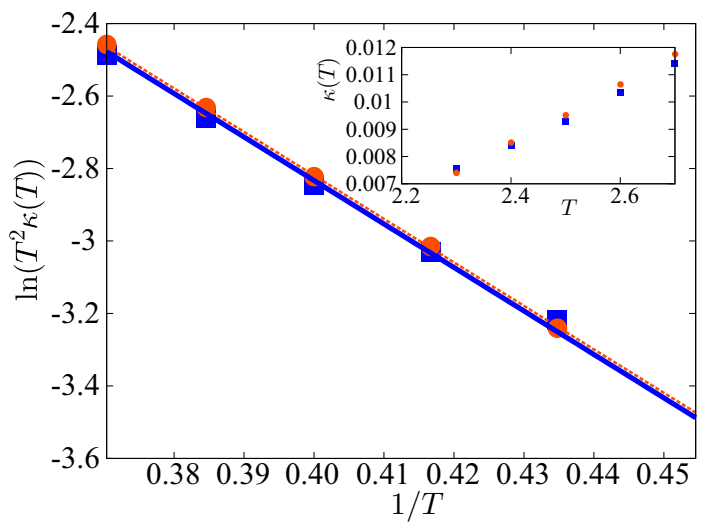

FIG. 4. Thermal conductivity $\kappa(T)$ of the system developed by the MC dynamics. For a system of size $10 \times 20 \times 20, \ln \left[T^{2} \kappa(T)\right]$ is plotted as a function of $1 / T$. Inset: plots of $\kappa(T)$ versus $T$. Orange circles and blue squares indicate the system with and without an interface, respectively. Orange dotted line and blue line are guides for the eye to show that $\kappa(T) \sim 1 / T^{2} \exp (-12 / T)$ at the hightemperature region.

of the diffusion constant of the interface parallel to heat flux is more interesting. The diffusion constant is estimated as follows. First, the position of an interface $z$ is defined by using magnetization $m=\left(L_{x} L_{y} L_{z}\right)^{-1} \sum_{i, j, k} \sigma_{i j k}$ as

$$
z=\frac{L_{z}\left(m+m_{0}\right)}{2 m_{0}}
$$

where we specified lattice points by the coordinates $(i, j, k)$, and $L_{x}, L_{y}$, and $L_{z}$ are the system size in each direction, and $m_{0}$ is spontaneous magnetization. Thus, if $m=-m_{0}$, the interface is at the bottom side $z=0$, and if $m=+m_{0}$, it is at the top side $z=L_{z}$. The diffusion constant $D$ is calculated from mean-square displacements of the interface position $z$ as

$$
\left\langle[z(t)-z(0)]^{2}\right\rangle=2 D t .
$$

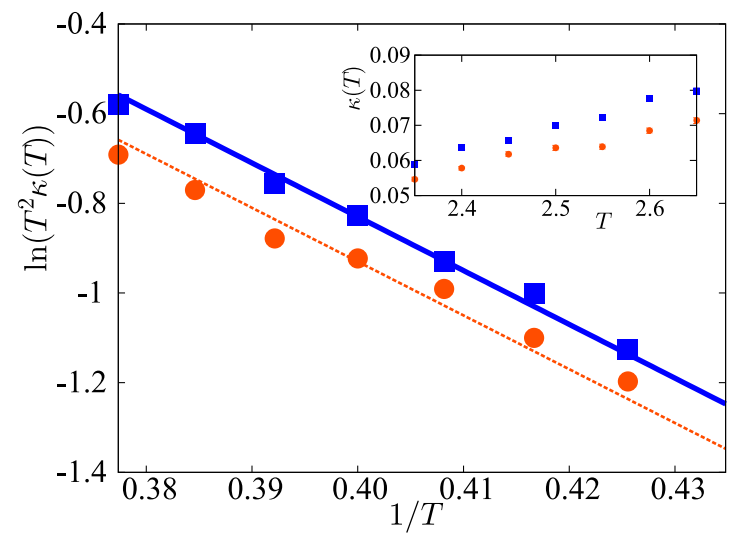

FIG. 5. Thermal conductivity $\kappa(T)$ of the system developed by the KSC dynamics. For a system of size $32 \times 32 \times 16, \ln \left(T^{2} \kappa(T)\right)$ is shown as a function of $1 / T$. Inset: plots of $\kappa(T)$ versus $T$. Orange circles and blue squares indicate the system with and without an interface, respectively. Orange dotted line and blue line are guides for the eye to show that $\kappa(T) \sim 1 / T^{2} \exp (-12 / T)$ at the hightemperature region.

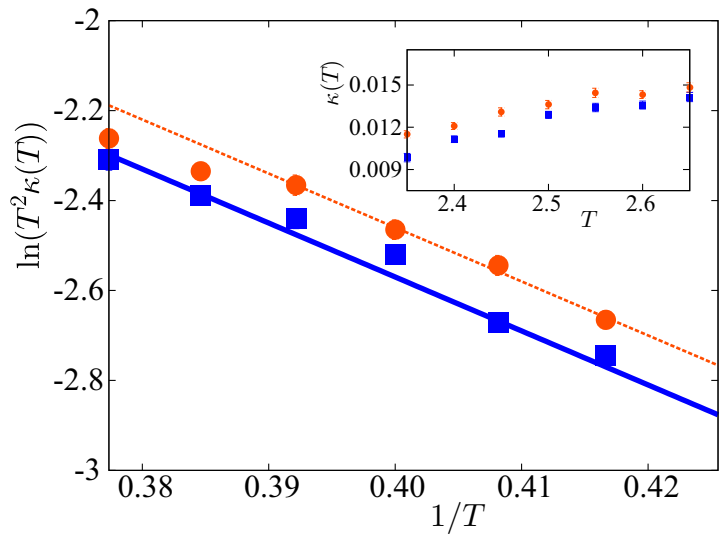

FIG. 6. Thermal conductivity $\kappa(T)$ of the system developed by the MC dynamics. For a system of size $32 \times 32 \times 16, \ln \left[T^{2} \kappa(T)\right]$ is shown as a function of $1 / T$. Inset: plots of $\kappa(T)$ versus $T$. Orange circles and blue squares indicate the system with and without an interface, respectively. Orange dotted line and blue line are guides for the eye to show that $\kappa(T) \sim 1 / T^{2} \exp (-12 / T)$ at the hightemperature region.

Note that temperature varies along an interface in the present setup. Thus, the roughness depends on the position on the interface. Notwithstanding that, we can obtain a diffusion constant that represents the interface motion as a whole.

Figure 7 shows logarithm of diffusion constant $D$ as a function of $1 / T$, which is obtained by using the $\mathrm{KSC}$ and the MC dynamics for equilibrium condition $T_{1}=T_{2}$. The magnitude of the diffusion constant is greater in the MC dynamics than in the KSC dynamics. However, the temperature dependence of the diffusion constant is similar in both the cases; that is, the diffusion constant is proportional to $\exp (-12 / T)$ above $T_{\mathrm{R}}^{\mathrm{eq}}$ and rapidly decreases below $T_{\mathrm{R}}^{\mathrm{eq}}$. This result implies that a smooth interface is difficult to move.

Figure 8 shows the logarithm of the diffusion constant $D$ obtained from the MC dynamics for the system under the temperature gradient. At the high-temperature region, the diffusion constant varies with temperature as $D \sim \exp (-12 / T)$

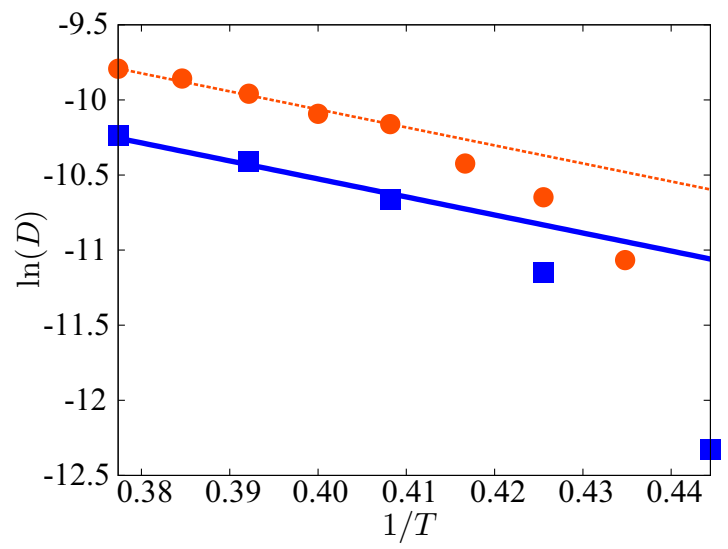

FIG. 7. For a system of size $32 \times 32 \times 16, \ln (D)$ is shown as a function of $1 / T$. Blue squares and orange circles indicate the KSC dynamics and the MC dynamics, respectively. Blue and black lines show $D(T) \sim \exp (-12 / T)$. 


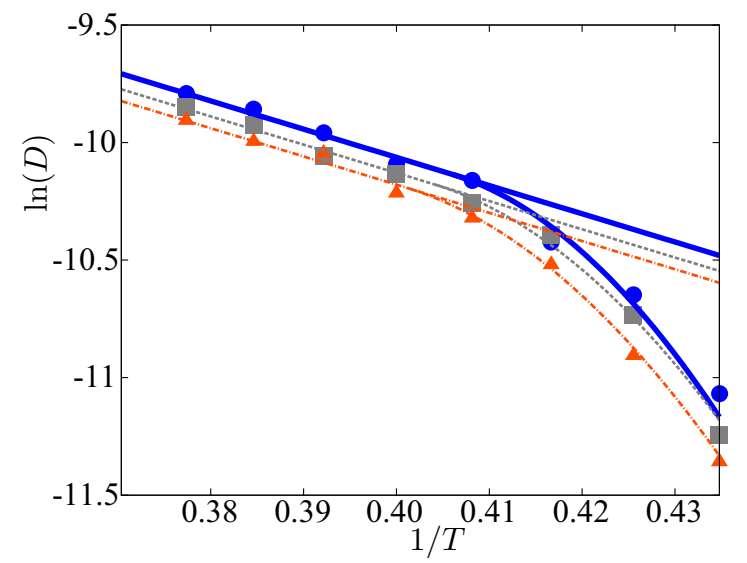

FIG. 8. For a system of size $32 \times 32 \times 16, \ln (D)$ is shown as a function of $1 / T$. Blue circles, gray squares, and orange triangles indicate the results of $\Delta T=0.0,0.3$, and 0.5 , respectively. Blue straight line, gray dotted straight, line and orange chain straight line straight lines indicate $D_{+}(T)=\exp (-12 / T+a)$, and blue curve, gray dotted curve, and orange chain curve indicate $D_{-}(T)=$ $\exp \left[-12 / T+a+A^{2}(1 / T-B)^{2}\right]$.

as in the equilibrium case, and it rapidly decreases below a certain crossover temperature $T_{\mathrm{X}}$. We estimate values of $T_{\mathrm{X}}$ in the following manner. First, we fit the numerical data by $D_{+}=\exp (-12 / T+a)$ in the high-temperature region, where $a$ is a fitting parameter. Next, we calculate the deviation from $D_{+}$as $\Delta=\ln D_{+}-\ln D$. As seen in Fig. $9, \sqrt{\Delta}$ is roughly proportional to $1 / T$ in the low-temperature region, where we fit the data by $\sqrt{\Delta}=A(1 / T-B)$ with parameters $A$ and $B$. That is, the diffusion constant in the low-temperature region is fitted by $D_{-}=\exp \left(-12 / T+a-A^{2}[1 / T-B)^{2}\right]$ as seen in Fig. 8. Then we identify the crossover temperature as $T_{\mathrm{X}}=$ $1 / B$. The obtained crossover temperature shows temperature dependence like $T_{\mathrm{X}} \sim T_{\mathrm{R}}^{\mathrm{eq}}+0.127 \Delta T$ as in Fig. 12 below.

In an equilibrium system, $T_{\mathrm{X}}$ and $T_{\mathrm{R}}^{\mathrm{eq}}$ are indistinguishable. Thus, the above result implies the possibility that the roughening transition temperature is shifted by heat conduction. To verify the implication, we estimate the roughening transition temperature in the system with heat conduction by using the

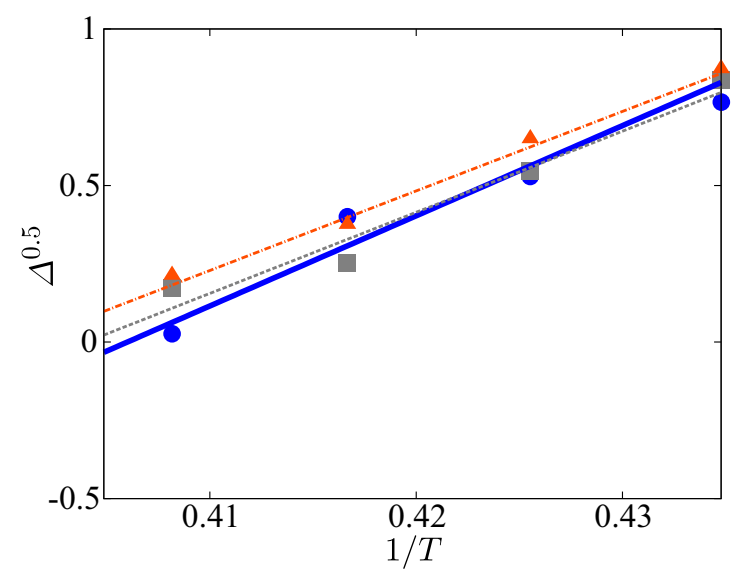

FIG. 9. Plot of $\sqrt{\Delta}$ versus $1 / T$. Blue circles, gray squares, and orange triangles indicate $\Delta T=0.0,0.3$, and 0.5 , respectively. Blue line, gray dotted line, and orange chain line are the estimated curves $D_{-}(T)$.

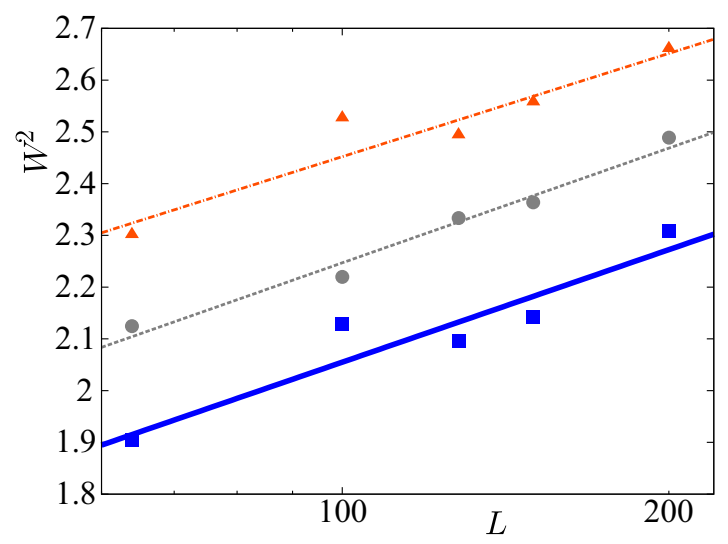

FIG. 10. Size dependence of $W^{2}$ for the high-temperature region $T>T_{\mathrm{R}}^{\mathrm{eq}}$ for the system of size $L \times L \times 20$ and the temperature difference $\Delta T=0.1$. Blue circles, gray squares, and orange triangles indicate $T=2.55,2.60$, and 2.65 , respectively. Blue line, gray dotted line, and orange chain line show $W^{2} \sim \ln L$.

width $W$ of an interface defined as [18]

$$
W^{2}=\frac{1}{\left(L_{x} L_{y}\right)^{2}} \sum_{i, j, k, l}\left\langle\left(h_{i j}-h_{k l}\right)^{2}\right\rangle,
$$

where $h_{i j}=1 /\left(2 m_{0}\right) \sum_{k} \sigma_{i j k}$ is the height of the interface at $(x, y)=(i, j)$. It is known that in equilibrium $W^{2}$ behaves as follows [8]:

$$
\begin{array}{cc}
W^{2} \sim\left[c_{1}+c_{2}\left(T-T_{\mathrm{R}}^{\mathrm{eq}}\right)^{1 / 2}\right] \ln L & \left(T>T_{\mathrm{R}}^{\mathrm{eq}}\right), \\
W^{2} \sim c_{3}+c_{4}\left(T_{\mathrm{R}}^{\mathrm{eq}}-T\right)^{-1 / 2} & \left(T<T_{\mathrm{R}}^{\mathrm{eq}}\right),
\end{array}
$$

where $c_{1}, c_{2}, c_{3}$, and $c_{4}$ are some constants.

We numerically calculated $W^{2}$ for the systems with a fixed temperature difference $\Delta T$, various average temperature $T$, and various system size $L$. As a result we found that there is a temperature $T_{\mathrm{R}}(\Delta T)$ such that if $T>T_{\mathrm{R}}(\Delta T), W^{2}$ behaves like Eq. (7) (Fig. 10), and if $T>T_{\mathrm{R}}(\Delta T)$, Eq. (8) is well satisfied (Fig. 11). Thus we call $T_{\mathrm{R}}(\Delta T)$ the nonequilibirum roughening transition temperature. Note that we always write

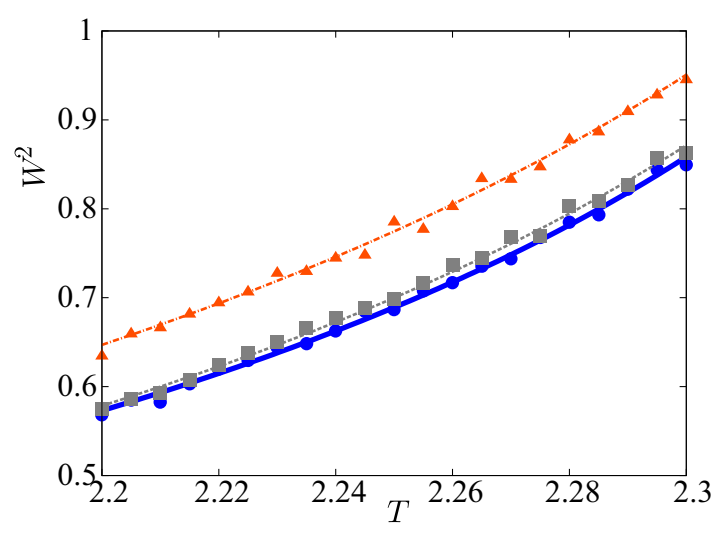

FIG. 11. Temperature dependence of $W^{2}$ for the low-temperature region $T<T_{\mathrm{R}}^{\mathrm{eq}}$ for the system of size $128 \times 128 \times 20$. Blue circles, gray squares, and orange triangles indicate $\Delta T=0.0,0.1$, and 0.3 , respectively. Blue, black, and orange lines show Eq. (8). 
the argument $\Delta T$ to distinguish it from the equilibrium roughening transition temperature $T_{\mathrm{R}}^{\mathrm{eq}}$.

The nonequilibrium roughening transition temperature $T_{\mathrm{R}}(\Delta T)$ thus obtained varies with $\Delta T$ like $T_{\mathrm{R}}(\Delta T) \sim T_{\mathrm{R}}^{\mathrm{eq}}+$ $0.118 \Delta T$. Figure 12 shows a comparison between $T_{\mathrm{X}}$ and $T_{\mathrm{R}}(\Delta T)$, which shows that $T_{\mathrm{X}}$ and $T_{\mathrm{R}}(\Delta T)$ agree with each other within error bars.

\section{SUMMARY AND DISCUSSION}

In this paper, we have numerically studied the relationship between the diffusion of an interface and heat conduction in the three-dimensional Ising model. We have examined how the dynamics and the arrangements of an interface affect heat conduction and the interface motion.

First, we investigated heat conduction in the two cases where the interface is perpendicular and parallel to the heat flux and with two kinds of dynamics: the KSC dynamics and the MC dynamics. We have found that whether an interface enhances heat conduction or not depends on dynamics. It is the case in the MC dynamics, but it is not in the $\mathrm{KSC}$ dynamics. In the case of an interface perpendicular to heat flux, the KSC dynamics yields a sudden decrease of thermal conductivity just below $T_{\mathrm{R}}^{\mathrm{eq}}$, while the MC dynamics does not. It shows that the MC dynamics is superior to the KSC dynamics for the use in low-temperature simulations.

Next, we computed the diffusion constant in the case where the interface is parallel to heat flux. The diffusion constant showed crossover in temperature dependence irrespective of dynamics. We estimated the crossover temperature $T_{\mathrm{X}}$, which agrees with the roughening transition temperature $T_{\mathrm{R}}^{\mathrm{eq}}$ in equilibrium and deviates from it in the presence of temperature gradient. It suggests some relationship between the roughness and the motion of the interface, but the functional form used for fitting is ad hoc and lacks any theoretical grounds. Then we calculated the width of the interface in the systems with a boundary-temperature difference $\Delta T$ and determined the nonequilibrium roughening transition temperature from their dependence on system size and temperature. The obtained nonequilibrium roughening transition temperature $T_{\mathrm{R}}(\Delta T)$

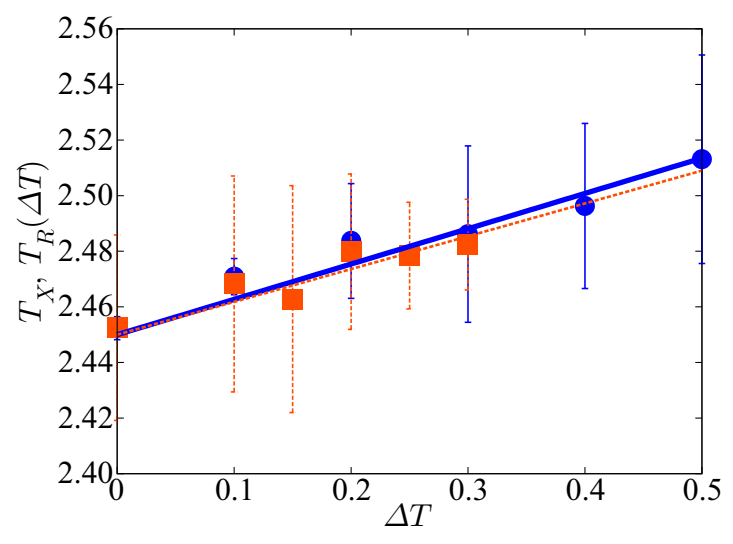

FIG. 12. $T_{\mathrm{X}}$ (blue circles) and $T_{\mathrm{R}}(\Delta T)$ (orange squares). Blue, black, and orange lines are fitted straight lines.

agrees with $T_{\mathrm{X}}$ within error bars, though the data are rather noisy. One may suspect that the result depends on dynamics. We carried out simulations with the Glauber dynamics with the same temperature profile as obtained in the MC dynamics and obtained almost the same result. Thus we do not consider that the behavior of $T_{\mathrm{X}}$ and $T_{\mathrm{R}}(\Delta T)$ comes from the peculiarity of the dynamics employed. The above results suggest the conjecture that heat conduction shifts the roughening-transition temperature. To our knowledge, this is the first time that such evidence has been found for the motion of the interface motion in the Ising model.

To establish this conjecture, we have to improve computational performance and develop theoretical considerations. The dynamics we employed in this study conserves local energy. In contrast to usual Monte Carlo dynamics, it is not as easy to accelerate or parallelize such dynamics. Thus we have been limited to modest system sizes. Improvements using, for example, the GPU are a future problem. In the classification by Hohenberg and Halperin [19], energy-conserving and magnetization-nonconserving dynamics like the KSC and MC dynamics are classified as Model C. A theoretical study of our findings based on Model $\mathrm{C}$ is desirable, because it means that the phenomena have a universal feature.

\section{ACKNOWLEDGMENTS}

Numerical computation in this work was carried out at the Yukawa Institute Computer Facility.
[1] J. P. Sethna, Statistical Mechanics: Entropy, Order Parameters, and Complexity (Oxford University Press, Oxford, 2006).

[2] T. O. E. Skinner, D. G. A. L. Aarts, and R. P. A. Dullens, Phys. Rev. Lett. 105, 168301 (2010).

[3] D. Hinzke and U. Nowak, Phys. Rev. Lett. 107, 027205 (2011).

[4] W. Jiang, P. Upadhyaya, Y. Fan, J. Zhao, M. Wang, L. T. Chang, M. Lang, K. L. Wong, M. Lewis, Y. T. Lin et al., Phys. Rev. Lett. 110, 177202 (2013).
[5] F. Schlickeiser, U. Ritzmann, D. Hinzke, and U. Nowak, Phys. Rev. Lett. 113, 097201 (2014).

[6] Y. Masumoto and S. Takesue, Phys. Rev. E 97, 052141 (2018).

[7] S. Takesue, T. Mitsudo, and H. Hayakawa, Phys. Rev. E 68, 015103(R) (2003).

[8] K. K. Mon, D. P. Landau, and D. Stauffer, Phys. Rev. B 42, 545 (1990). 
[9] A.-L. Barabási and H. E. Stanley, Fractal Concepts in Surface Growth (Cambridge University Press, Cambridge, 1995).

[10] A. V. Babkin, D. B. Kopeliovich, and A. Ya. Parshin, Sov. Phys. JETP 62, 1332 (1985).

[11] R. J. Glauber, J. Math. Phys. 4, 294 (1963).

[12] M. Creutz, Ann. Phys. 167, 62 (1986).

[13] K. Saito, S. Takesue, and S. Miyashita, Phys. Rev. E 59, 2783 (1999).

[14] G. Y. Vichniac, Physica D 10, 96 (1984).
[15] M. Casartelli, N. Macellari, and A. Vezzani, Eur. Phys. J. B 56, 149 (2007).

[16] E. Agliari, M. Casartelli, and A. Vezzani, J. Stat. Mech. (2009) P07041.

[17] A. M. Ferrenberg, J. Xu, and D. P. Landau, Phys. Rev. E 97, 043301 (2018).

[18] M. Hasenbusch, M. Marcu, and K. Pinn, Physica A 208, 124 (1994).

[19] P. C. Hohenberg and B. I. Halperin, Rev. Mod. Phys. 49, 435 (1977). 